$63^{\text {ème }}$ Congrès de la SFCO, 03009 (2015)

DOI:10.1051/sfco/20156303009

(C) Owned by the authors, published by EDP Sciences, 2015

\title{
La résection apicale avec utilisation d'IRM et du microscope : taux de succès pour 122 cas sur un suivi de 24 mois
}

\author{
Wdowik S, Godard A, Limbour P \\ CHU Rennes, 2 rue Henri Le Guilloux, 35000 Rennes
}

Introduction

La chirurgie apicale endodontique est une méthode de traitement d'une dent présentant une lésion apicale, qu'un (re)traitement canalaire conventionnel orthograde ne parvient pas à traiter. Le risque lié à la dépose d'éléments prothétiques (bridge, couronne, reconstitution corono-radiculaire avec ancrage canalaire) peut également indiquer la chirurgie apicale.

Les résultats ont été améliorés par le perfectionnement de nouveaux matériaux (ciment super-EBA, IRM, MTA, Biodentine) et instruments (loupes binoculaires, ultrasons, microinstruments): le microscope opératoire est notamment de plus en plus utilisé.

Le but de cette étude rétrospective est d'évaluer le taux de succès de la chirurgie apicale sous microscope opératoire et avec une obturation rétrograde à l'IRM, telle qu'elle est réalisée en pratique clinique, c'est-àdire sans sélection du type de dent.

Matériels et méthode

175 dents pour 133 patients présentant une lésion apicale, ont été traitées par chirurgie apicale dans le service de Chirurgie Buccale du CHU de Rennes, de juillet 2007 à avril 2011. Le suivi post-opératoire a été réalisé pendant deux ans.

La procédure était standardisée : même chirurgien, même technique (ultrasons, IRM, sous microscope opératoire).

Une dent n'était exclue que si une fêlure/fracture non traitable était diagnostiquée, si elle avait subi une chirurgie apicale antérieure, ou en cas d'absence de suivi. La qualité de l'obturation orthograde n'a pas été un facteur de décision pour réaliser la chirurgie, toutes les dents ont été inclues dans l'étude.

Pour chaque dent, des radiographies pré et post-opératoires, puis à chaque rendez-vous de suivi, ont été réalisées avec un angulateur, selon la technique des plans parallèles.

Deux praticiens-observateurs calibrés ont examiné les radiographies, deux fois chacun à 15 jours d'intervalle. La symptomatologie a été rapportée lors des différents contrôles.

Les résultats pour chaque dent ont été classés dans 4 catégories : guérison complète, guérison incomplète, guérison incertaine, absence de guérison.

Résultats

122 dents pour 94 patients ont été inclues : 41 incisives/canines, 20 prémolaires, 61 molaires. Le pourcentage de succès est $78,70 \%$. Un succès est obtenu pour 96 dents, dont 64 guérisons complètes avec réossification, lamina dura, trabéculation osseuse, recouvrement d'un mince et régulier ligament alvéolo-dentaire, condensation et densité de l'obturation optimale. 32 dents présentent une guérison incomplète avec une légère image radioclaire mais sans symptomatologie. 4 dents sont classées en guérison incertaine : légère symptomatologie et persistance d'une image radioclaire. 22 dents sont classées en échec total : continuité de la symptomatologie initiale, persistance d'une lésion radioclaire égale ou augmentée, discontinuité de la lamina dura.

This is an Open Access article distributed under the terms of the Creative Commons Attribution License 4.0, which permits unrestricted use, distribution, and reproduction in any medium, provided the original work is properly cited. 


\section{Discussion}

Le but était de représenter la réalité clinique de la pratique chirurgicale : toutes les dents adressées par les praticiens ont donc été inclues dans l'étude, sans égard pour la qualité de l’obturation orthograde, ni pour la position de la dent sur arcade. $50 \%$ des dents sont des molaires, or il est reconnu que le pronostic de chirurgie apicale sur ces dents est plus réservé. Le taux de succès est donc élevé compte tenu des critères d'inclusion. La visibilité parfaite du champ opératoire est une des clés de la réussite du traitement. La technique « microscope/IRM » a démontré son efficacité.

Nom et adresse du conférencier

\section{Selya WDOWIK}

CHU Rennes

2 rue Henri Le Guilloux

35000 Rennes (France)

selya.wdowik@hotmail.fr 